\title{
Vaccine Hesitancy and Misinformation About Clinical Trials
}

\author{
Bhattacharya Surajit ${ }^{1} \mathbb{D}$
}

Received: 16 June 2021 / Accepted: 17 June 2021 / Published online: 24 June 2021

(c) Association of Surgeons of India 2021

In our attempt to vaccinate all, vaccine hesitancy is turning out to be a huge problem in our country. If we fail to vaccinate all and pockets of unvaccinated people remain, then coronavirus can stay in them, mutate, and reinfect others. Community leaders, religious and political, need to galvanize public opinion in favor of vaccine as healthcare workers can, at best, only partly fill up this leadership vacuum. Any misinformation, misgivings, and apprehensions expressed by our community leaders will set off a domino effect of vaccine non-compliance in our society. Drugs Controller General of India (DCGI) approved two vaccines Covishield and Covaxin for "emergency" use on January 3, 2021. However, DCGI did so even as Covaxin's third phase clinical trials were just getting started. This initiated the ongoing misinformation infodemic about our vaccines. For years, a central but false tenet of the anti-vaccine lobby has been that vaccines are not adequately tested for safety. Our countrymen will be used as guinea pigs they said and thus sowed the seeds of disbelief in the very early days of the pandemic. Today, we are seeing the results of this mischievous propaganda, and doctors are being harassed and chased away by irate and misinformed villagers, and our vaccination drive is failing to keep pace. It therefore becomes imperative to understand how vaccine trial takes place, its format, its method and its timeline, and why this approval for emergency use was grancted by DGCI.

An "average vaccine" requires a 10.7-year timeline beginning with the pre-clinical phase, whereas COVID-19 vaccine program completed clinical testing in less than a year. However, the research on COVID-19 vaccines did not first begin in 2020 but instead built on a decade of previous research on coronaviruses, leading to proof-of-concept for the spike protein as a lead vaccine target. In both the USA and internationally, phase 3 trials were well-powered studies of 30,000 to 60,000 human volunteers, equivalent to other

Bhattacharya Surajit

surajitbh@yahoo.co.in

1 Sahara Hospital, Lucknow, India large vaccine clinical trials required to license vaccines. So the vaccines available to us today are not half cooked products but just as well researched as any other vaccine.

From my experience of being a member of a reputed institutional ethics committee, let me briefly tell you about how clinical trials for new drugs or vaccines are conducted. These trials and their individual phases are a very important part of clinical research because they allow the safety and effectiveness of new drugs to be properly assessed before being approved for use in the general public.

\section{Pre-clinical Research}

Even before doing a clinical trial, investigators conduct preclinical research using human cell cultures or animal models to study whether a new medication or vaccine is toxic to a small sample of human cells in a laboratory.

\section{Phase 0}

This phase of a clinical trial is done with a very small number of people, usually fewer than 15 . Investigators use a very small dose of medication to make sure it isn't harmful to humans before they start using it in higher doses for later phases.

If the medication acts differently than expected, the investigators will likely do some additional pre-clinical research and animal studies before deciding whether to continue the trial.

\section{Phase I}

During this phase, the investigators spend several months looking at the effects of the medication on about 20 to 80 people who have no underlying health conditions.

This phase aims to figure out the highest dose humans can take without serious side effects. They also look at the best 
way to administer the drug, such as orally, intravenously, or topically.

\section{Phase II}

This phase involves several hundred participants who are living with the condition that the new medication is meant to treat. They're usually given the same dose that was found to be safe in the previous phase. This phase can stretch from several months to years to understand the efficacy as well as the side effects.

\section{Phase III}

This phase of a clinical trial usually involves up to 3,000 participants who have the condition that the new medication is meant to treat. Trials in this phase can last for several years.

The purpose of phase III is to evaluate how the new medication works in comparison to existing medications for the same condition. To move forward with the trial, investigators need to demonstrate that the medication is at least as safe and effective as existing treatment options. Needless to say that in case of a vaccine against COVID19, there were no existing vaccines for comparison, and all double-blind trials were against a placebo.

Phase III trials are usually double-blind, which means that neither the participant nor the investigator knows which medication the participant is taking. This helps to eliminate bias when interpreting results.

\section{Phase IV}

Phase $1 \mathrm{~V}$ clinical trials happen after the FDA or Drug Controller General of a country has approved medication. This phase involves thousands of participants and can last for many years. Investigators use this phase to get more information about the medication's long-term safety, effectiveness, and any other benefits.
According to the FDA, only $70 \%$ of trial drugs move up from phase I to phase II and 33\% of them from phase II to phase III. Finally, 25 to $30 \%$ of trials in phase III move to phase IV.

So now that you have a fairly good idea of the usual timeline of a drug trial, do you think we had the luxury of following the classical timeline with the COVID vaccines? How many lives would have been lost if we would have waited for a classical phase III trial?

So what can we surgeons do? Now as practice is feeble and we have some time to spare, we must also roll up our sleeves and actively help. I am sure all of you have noticed that across the spectrum of social media, miscreants and fakers are invariably more innovative and savvy. Our educated and intelligent members must help in countering this tidal wave of misinformation about the disease, the virus, and vaccines and ensure that consistent and factually accurate messages are being distributed. Each of us must navigate their own social media circles and offer critical tools, genuine resources and correct advice to the masses.

We must encourage people to get vaccinated and fight vaccine hesitancy. Let no one be complacent - we are battling multiple enemies on multiple fronts, and we cannot see them - ignorance, mischief, misinformation, false narratives, and fake news are everywhere. Even the information about what we do know seems to change as quickly as we hear it. When this is an ever-changing story that is difficult for even us professionals to follow, then how can amateur lay people be expected to do any better? In these dangerous hours, days, weeks, and months of a still-evolving global health crises, the lay public needs hand holding, and we must do our bit. We are considered the cream of society, and it is our duty, nay our responsibility and obligation, to play our part with all our strength and determination.

Publisher's Note Springer Nature remains neutral with regard to jurisdictional claims in published maps and institutional affiliations. 